\title{
Secreción de prolactina en hombres nativos de las grandes alturas
}

\author{
Prolactin secretion in high altitude natives
}

\author{
José Solís J. ${ }^{1,2}$, Róger Guerra-García R. ${ }^{3}$, S. Acosta ${ }^{3}$ y Juan Hurtado ${ }^{4}$ \\ 'Profesor Principal de Medicina, Universidad Peruana Cayetano Heredia. \\ ${ }^{2}$ Jefe del Servicio de Endocrinología del Hospital Nacional Arzobispo Loayza. \\ ${ }^{3}$ Profesor Emérito de la Universidad Peruana Cayetano Heredia. \\ ${ }^{4}$ Médico Asistente del Hospital Nacional Arzobispo Loayza.
}

\begin{abstract}
Resumen
Objetivos: Determinar el efecto de la hipoxia crónica sobre la secreción de prolactina. Diseño: Estudio comparativo. Institución: Servicio de Endocrinología, Hospital Nacional Arzobispo Loayza, Lima, Perú. Participantes: Hombres jóvenes nativos de Lima y Cerro de Pasco. Intervenciones: Se estudió en forma dinámica tres grupos de hombres jóvenes normales de edad (X24a) y estado nutricional comparable, nativos de diferentes altitudes: 150 m.s.n.m. (Lima, $N=10$ ), 3000 m.s.n.m. (Tarma, $N=10$ ), 4200 m.s.n.m. (Cerro de Pasco, $n=11$ ); y tres pacientes con mal de montaña crónico (MMC) nativos de Cerro de Pasco; todos clínicamente eutiroideos y sin bocio. En cada sujeto se determinó por RIA los niveles séricos basales de PRL y su respuesta. Principales medidas de resultados: Diferencia de niveles séricos de prolactina y su respuesta máxima a la hormona liberadora de tirotropina en sujetos a nivel del mar y en la altura. Resultados: Los niveles séricos basales de prolactina (PRL) y su respuesta máxima a la hormona liberadora de tirotropina (TRH) fueron significativamente menores en los nativos de altura en comparación con los del nivel del mar. La causa subyacente sería la hipoxia crónica, pudiendo estar implicados un incremento de la actividad dopaminérgica del sistema túbero - infundibular o una alteración a nivel del receptor o posreceptor del lactotrofo en los nativos de altura. Este hecho puede ser un mecanismo de adaptación para la conservación de la fertilidad en los hombres nativos de las grandes alturas. El escaso número de pacientes con mal de montaña crónico no permitió obtener resultados concluyentes, pero se observó persistencia de la menor secreción de prolactina, con una respuesta más tardia a la TRH. Se requiere más investigaciones en pacientes con MMC, para definir estas observaciones. Conclusiones: Los niveles séricos basales de prolactina y su respuesta máxima a la hormona liberadora de tirotropina fueron significativamente menores en los nativos de altura en comparación con los del nivel del mar.
\end{abstract}

Palabras clave: Prolactina, secreción de prolactina, hombres normales de grandes alturas, hipoxia crónica.

Abstract

Objectives: To determine the effect of chronic hypoxia on prolactin (PRL) secretion. Design: Comparative study. Setting: Endocrinology service, Hospital Nacional Arzobispo Loayza, Lima, Peru. Participants: Native young men from Lima and Cerro de Pasco. Interventions: Three groups of normal young men age (X24a) with comparable nutritional status, natives from different altitudes: 150 m.a.s.l. (Lima, $\mathrm{N}=10), 3000$ m.a.s.I. (Tarma, $\mathrm{N}=10$ ), 4200 m.a.s.I. (Cerro de Pasco, $n=11$ ); and three patients with chronic mountain disease (CMD) natives from Cerro de Pasco were studied. All subjects were euthyroid and without goiter. RIA determinations of PRL basal serum levels and PRL response to thyrotrophin releasing hormone (TRH) $200 \mu \mathrm{g} \mathrm{I.V.} \mathrm{were} \mathrm{carried} \mathrm{out} \mathrm{on} \mathrm{each} \mathrm{subject.} \mathrm{Main} \mathrm{outcome} \mathrm{measures:}$ Difference of serum prolactin levels and highest response to thyrotrophin releasing hormone in subjects at sea level and at high altitude. Results: Prolactin basal serum levels and the highest response to TRH were significatively less $(P<0.05)$ in high altitude natives when compared to those at sea level. Underlying cause would be chronic hypoxia including either tubero-infundibular dopaminergic system activity increase or lactotrophs' receptor or post-receptor alteration in high altitude natives. This may be an adaptation mechanism for fertility preservation in high altitude natives. The few patients with chronic mountain disease studied did not allow concluding results, but persistence of less prolactin secretion was observed with late response to TRH. Further investigations in patients with MMD are required to define these observations. Conclusions: Prolactin basal serum levels and highest response to thyrotropin releasing hormone were significatively less in high altitude natives compared with those at sea level.

Keywords: Prolactin, prolactin secretion, high altitude natives, chronic hypoxia.

An Fac med. 2011;72(1):45-50

\section{INTRODUCCIÓN}

En el Perú, aproximadamente el 30\% de nuestra población habita en las grandes alturas (3 000 o más metros sobre el nivel del mar), en donde se dan condiciones de hipoxia crónica y temperaturas bajas.
En la exposición aguda a las grandes alturas (hipoxia aguda) se ha descrito disminución de la fertilidad en animales y humanos ${ }^{(1)}$; también, aumento de triiodotironina (T3) y tiroxina (T4), incremento de ligazón de globulina fijadora de tiroxina (TBG) y discreta disminución de la respuesta de tirotro- pina (TSH) a la hormona liberadora de tirotropina (TRH) ${ }^{(2-4)}$. En cambio, en la exposición crónica a las grandes alturas (hipoxia crónica) tanto la fertilidad como la función tiroidea están preservadas en humanos y animales ${ }^{(1,5-8)}$.

En hombres normales, los niveles séricos de prolactina (PRL) guardan rela- 
ción entre otras cosas con la función reproductiva y la función tiroidea. Se ha determinado que en hombres y mujeres nativos de altura, los niveles basales de PRL se encuentran significativamente más bajos en comparación con los nativos del nivel del mar ${ }^{(1,9,10)}$.

El propósito del presente trabajo fue evaluar el efecto de la exposición crónica a grandes alturas sobre la secreción de PRL, tanto en su estado basal como en respuesta al estímulo de tiroliberina (TRH), en sujetos normales; para ello, hemos estudiado dos grupos de hombres jóvenes nativos de Tarma (3000 m.s.n.m.) y Cerro de Pasco (4 200 m.s.n.m.), en comparación con un grupo control de nativos o residentes de Lima (150 m.s.n.m.). Adicionalmente, se ha determinado el nivel de secreción del PRL en tres pacientes con Enfermedad de Monge, nativos de Cerro de Pasco.

\section{MÉTODOS}

Se realizó un ensayo clínico de intervención, no controlado ni aleatorizado. Se consideró tres grupos de hombres normales y sin bocio, nativos o residentes por más de 10 años, en tres altitudes geográficas: 150 m.s.n.m. (Lima, $\mathrm{n}=10$ sujetos); 3000 m.s.n.m. (Tarma, $\mathrm{n}=10$ sujetos) y 4200 m.s.n.m. (Cerro de Pasco, $n=11$ sujetos). La edad promedio fue similar en los tres grupos $(\mathrm{M} \pm \mathrm{DS} 25,8 \pm 3,23,3 \pm 3,24,2 \pm 13,9$, respectivamente). Su estado nutricional fue bueno y similar en los tres grupos; así, tanto el índice de masa corporal como el hematocrito fueron similares en los grupos de estudio, no existiendo diferencia significativa. Asimismo, se ha estudiado tres pacientes con mal de montaña crónico (Enfermedad de Monge), nativos de Cerro de Pasco, cuyas características generales se da en la tabla 1.

Todos ellos eran clínicamente eutiroides y no tenían bocio. El diagnóstico de Enfermedad de Monge se estableció en base a una hipereritremia, electrocardiograma (ECG) con evidencia de hipertrofia ventricular derecha, ausencia de antecedentes de enfermedad cardiopulmonar, rayos $\mathrm{X}$ de pulmones normales y ninguno de ellos era minero.

En cada sujeto de estudio se hizo un examen clínico completo. Luego, estando en ayunas, se les tomó dos muestras basales de sangre, con intervalos de 20'. Inmediatamente después se les inyectó vía endovenosa $200 \mu \mathrm{g}$ de TRH en bolo (marca Roche) y se tomó muestras de sangre a los 15, 30, 60, 90, 120 y $180^{\prime}$ post - TRH. Posteriormente, las muestras fueron centrifugadas y los sueros congelados a $-20^{\circ} \mathrm{C}$ hasta su proceso. En la mezcla de alícuotas iguales de los sueros basales, se hizo determinaciones hormonales de PRL, T3, T4 y TSH; en todas las demás muestras se determinó PRL y TSH, para estudiar su respuesta a TRH. En cada sujeto se determinó la excreción urinaria de iodo (EUI) en 24 horas.

Las determinaciones hormonales fueron hechas por radioinmunoanálisis; los métodos usados han sido previamente descritos ${ }^{(11-14)}$. Las muestras fueron procesadas por duplicado, incluyendo todas las muestras de cada pa- ciente en un mismo ensayo. El sistema usado fue el de doble antisuero en fase líquida. Los coeficientes de variación (C.V.) intra e interensayo estuvieron en 5 y 8 por ciento, respectivamente.

Para las comparaciones entre los grupos se ha usado la prueba $t$ de student para muestras no pareadas ${ }^{(15)}$.

Las iodurias fueron determinadas por el método descrito por Benotti $\mathrm{S}$ y Benotti ${ }^{(16)}$.

El trabajo fue aprobado por la Universidad Peruana Cayetano Heredia y recibió apoyo de la Organización Internacional de Energía Atómica (OIEA). Los sujetos de investigación firmaron un documento aceptando su participación en el proyecto.

\section{RESULTADOS}

Los niveles basales de prolactina fueron significativamente menores en Cerro de Pasco $(\mathrm{P}<0,05)$ y Tarma $(\mathrm{P}<0,01)$ cuando se los comparó con el grupo de Lima (tabla 2, figura 1) y no hubo diferencia estadísticamente significativamente entre los grupos de Cerro de Pasco y Tarma. Todos los varones normales estudiados fueron eutiroideos clínica y bioquímicamente (tabla 3).

La respuesta de PRL a TRH en cuanto a su forma fue similar en los tres grupos, el $\Delta$ máximo de PRL post TRH ( $\Delta$ max $\mathrm{PRL}=$ pico más alto de respuesta - valor basal) se produjo generalmente a los $15^{\prime}$ post - TRH, en todos los grupos (figura 2) y fue significativamente menor en Tarma $(\mathrm{P}<0,05)$ y Cerro de Pasco $(\mathrm{p}<0,05)$, cuando se los comparó

Tabla 1. Sujetos con mal de montaña crónico. Características generales.

\begin{tabular}{|c|c|c|c|c|c|c|c|c|c|}
\hline Sujeto & Edad (años) & Estatura (m) & Peso (kg) & HTC (\%) & $\begin{array}{c}\text { Ciudad } \\
\text { nacimiento }\end{array}$ & $\begin{array}{c}\text { Ciudad } \\
\text { procedencia }\end{array}$ & Ocupación & $\begin{array}{l}\text { Enf. cardio } \\
\text { pulmonar }\end{array}$ & Bocio \\
\hline E.P. & 31 & 1,64 & 63 & 67 & C.P. & C.P. & Conserje & NO & NO \\
\hline A.H. & 41 & 1,63 & 63,6 & 69 & C.P. & C.P. & Herrero & NO & NO \\
\hline R.S. & 42 & 1,65 & 74 & 63 & C.P. & C.P & Comerc & NO & NO \\
\hline$X \pm$ D.S. & $38 \pm 6,1$ & $1,64 \pm 0,01$ & $66,9 \pm 6,1$ & $66,3 \pm 3,1$ & & & & & \\
\hline
\end{tabular}


Tabla 2. Efecto de la altura en la respuesta de prolactina a $200 \mu \mathrm{g}$ de TRH E.V. en hombres normales.

\begin{tabular}{|c|c|c|c|c|c|c|c|c|}
\hline \multirow{2}{*}{$\begin{array}{l}\text { Tiempo post TRH E.V. } \\
200 \mu \mathrm{g} \text { (minutos) }\end{array}$} & \multicolumn{8}{|c|}{ PRL ng/mL } \\
\hline & $0^{\prime}$ & $15^{\prime}$ & 30 & $60^{\prime}$ & $90^{\prime}$ & $120^{\prime}$ & $180^{\prime}$ & Max de PRL \\
\hline $\begin{array}{l}\text { Lima }(N=10) \\
150 \text { m.s.n.m. } \\
\quad X \pm \text { E.S. }\end{array}$ & $11,6 \pm 1,7$ & $64,5 \pm 17$ & $45,0 \pm 10,6$ & $33,0 \pm 8,1$ & $20,8 \pm 4,4$ & $14 \pm 2,6$ & $12,7 \pm 2$ & $62,7 \pm 16$ \\
\hline $\begin{array}{c}\text { Tarma }(N=10) \\
3000 \text { m.s.n.m. } \\
X \pm \text { E.S. }\end{array}$ & $5,5 \pm 0,4$ & $30,6 \pm 0,9$ & $24,5 \pm 2,8$ & $12,9 \pm 1,2$ & $9,7 \pm 0,8$ & $6,8 \pm 0,6$ & $4,9 \pm 0,4$ & $29,5 \pm 5$ \\
\hline $\begin{array}{l}\text { Cerro de Pasco }(\mathrm{N}=11) \\
\begin{array}{c}4200 \text { m.s.n.m. } \\
X \pm \text { E.S. }\end{array}\end{array}$ & $7,7 \pm 1$ & $32,9 \pm 4,8$ & $29,3 \pm 2,9$ & $21,9 \pm 2,7$ & $12,8 \pm 2,5$ & $10,9 \pm 2,5$ & $8,5 \pm 1,8$ & $28,8 \pm 4,3$ \\
\hline
\end{tabular}

PRL 0': Lima - Tarma P<0,01, Lima - Cerro de Pasco P<0,05. Max PRL: Lima - Tarma P< 0,05, Lima - Cerro de Pasco P<0,05. Rango normal en Lima: PRL: 3 a 20 ng/mL.

con el grupo de Lima (figura 3, tabla 2); no hubo diferencia estadísticamente significativa entre los grupos de Cerro de Pasco y Tarma.

Los resultados de las determinaciones basales de T3, T4, TSH y PRL, así como la respuesta de PRL a un estimulo de $200 \mu \mathrm{g} / \mathrm{EV}$ de TRH de tres pacientes con mal de montaña crónico, en comparación con el grupo de nativos normales del mismo lugar (Cerro de Pasco), son mostrados en la tabla 4.

En razón del escaso número de pacientes con mal de montaña crónico estudiados, no es posible hacer comparaciones estadísticas. Se aprecia, sin embargo, en lo relacionado a la secreción de prolactina, en comparación con el grupo de nativos normales, que los valores basales tienen tendencia a ser menores y la respuesta TRH en los tres pacientes con mal de montaña crónico es de magnitud similar a la de los nativos normales, pero más tardía; el $\Delta$ max PRL ocurrió entre los 30' y $60^{\prime}$ en los pacientes con mal de montaña crónico y en los nativos normales generalmente a los $15^{\prime}$ post-TRH.

\section{DISCUSIÓN}

Con relación a la secreción de prolactina en varones jóvenes normales de grandes alturas, los valores basales de prolactina (PRL) fueron significativamente menores en Cerro de Pasco $(\mathrm{P}<0,05)$ y Tarma $(\mathrm{P}<0,01)$ que en el grupo control de Lima, pero no hubo diferencia estadísticamente significativa entre los grupos de Tarma y Cerro de Pasco. Estos datos ya fueron reportados por nosotros en $1981^{(9,17)}$ y confirmados por otros autores ${ }^{(1,15)}$.

La respuesta de PRL a THR en los grupos de altura fue similar en forma, pero significativamente de menor magnitud que la del nivel del mar, el $\Delta$ max de PRL se produjo generalmente a los $15^{\prime}$ post - TRH en todos los grupos (figura 4).

Nuestros estudios demuestran que hay un cambio de la secreción de prolactina en el nativo de altura, en re- lación con el nativo del llano; para la explicación de este evento no es posible plantear una alteración en la secreción de TRH, ya que la secreción de TSH en el nativo de altura es similar a la del hombre del nivel del mar, como lo demostramos con anterioridad ${ }^{(18)}$.

El compromiso negativo de la secreción de prolactina que observamos en el nativo de altura no es un hallazgo aislado. Un compromiso similar se ha descrito para la secreción de la LH post-GnRh y post citrato de clomifeno ${ }^{(19,20)}$. Esto nos hace plantear la posibilidad de que pudiera darse una mayor actividad dopaminérgica en el sistema

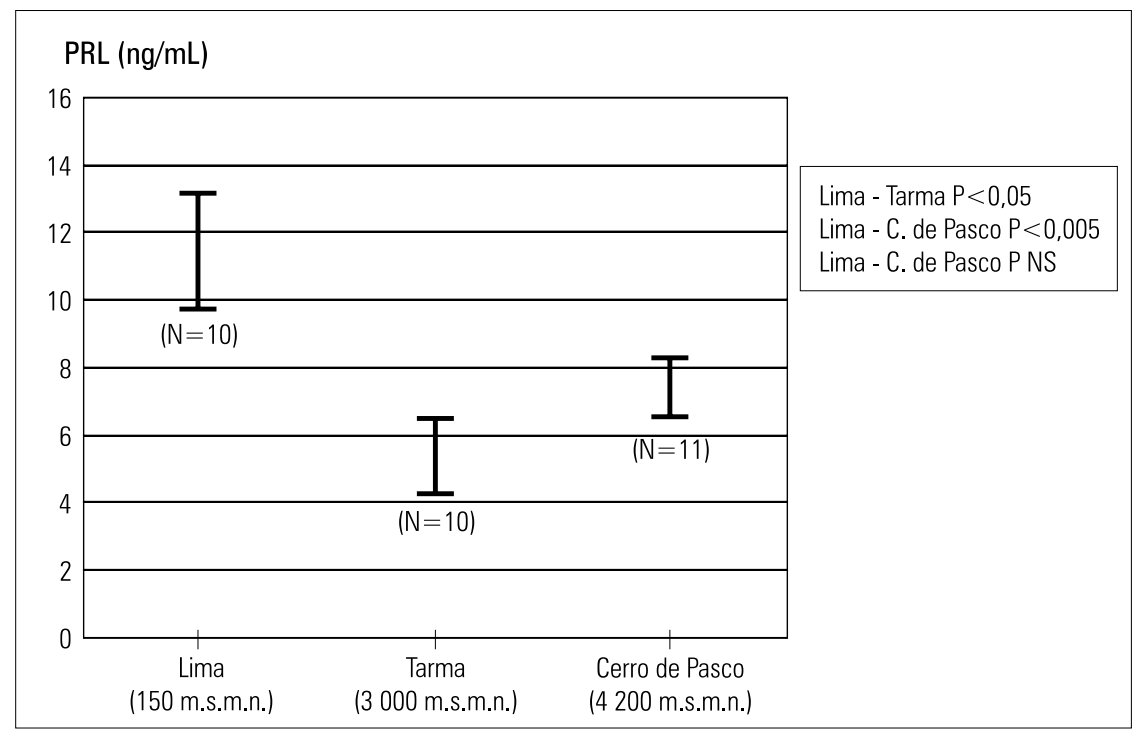

Figura 1. Valores basales de prolactina en hombres a nivel del mar y a grandes alturas. 
túbero-infundibular del nativo de altura como posible explicación del compromiso descrito de la secreción de prolactina; es conocido que el incremento de la actividad del sistema dopaminérgico túbero-infundibular puede inhibir la secreción de prolactina y de GnRh y por ende la de $\mathrm{LH}^{(21-25)}$. El factor subyacente de esta supuesta mayor actividad dopaminérgica del sistema túbero - infundibular sería la hipoxia crónica.

También es posible plantear como explicación alternativa de la menor secreción de prolactina post-TRH del nativo de altura la posible existencia de algún cambio a nivel del receptor o post-receptor de los lactotrofos ocasionado por la hipoxia crónica, ya que se ha reportado en varones nativos de grandes alturas moderadas (3 395 m.s.n.m.) igual sensibilidad de la hipófisis a la administración en bolo de GnRh ${ }^{(26)}$ y similar respuesta de $\mathrm{LH}$ al citrato de clomifeno en comparación con varones del nivel del mar ${ }^{(27)}$.

La menor secreción de PRL observada en el hombre de grandes alturas podría ser un mecanismo de adaptación para conservar la fertilidad en condiciones de hipoxia crónica.

Respecto a la secreción de prolactina en pacientes con mal de montaña crónico, el escaso número de pacientes estudiados (tres) no nos permite obtener conclusiones. Sin embargo, en los pacientes con mal de montaña crónico

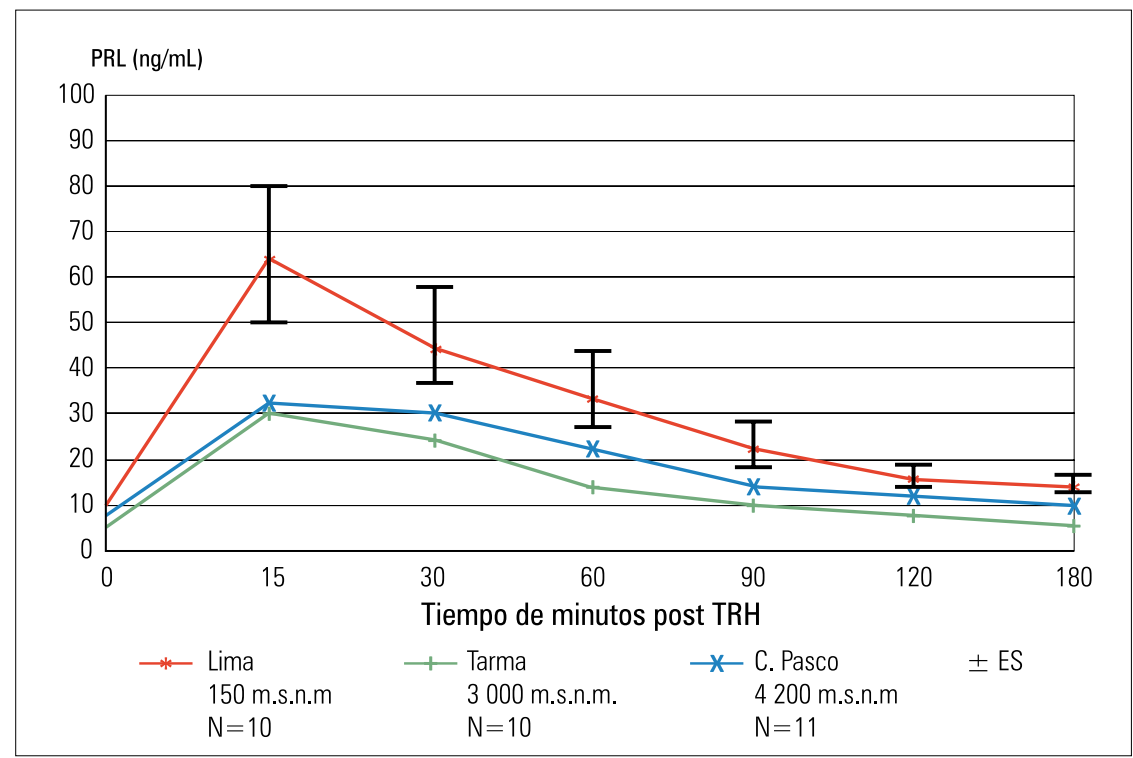

Figura 2. Efecto de la altura en la respuesta de prolactina a $200 \mu \mathrm{g}$ de TRH EV en hombres normales.

en comparación con los nativos normales de grandes alturas se observa una tendencia a menores valores basales de prolactina y una respuesta al TRH similar en magnitud pero más tardía, lo cual nos sugiere un mayor compromiso de la secreción de prolactina que el observado en el nativo normal de altura. Dado que en los pacientes con mal de montaña crónica hay un grado mayor de hipoxia, esto aboga a favor de que la hipoxia crónica es el factor causal del compromiso de la secreción de prolactina post- TRH observado en el nativo normal de altura. Estos hallazgos re- quieren de mayores observaciones para su confirmación, lo cual constituye una meta para futuras investigaciones.

En conclusión, en los hombres jóvenes normales nativos de las grandes alturas, en comparación con los del nivel del mar, los niveles séricos basales de prolactina y su respuesta máxima al TRH endovenoso estuvieron disminuidos. La causa sería la hipoxia crónica y como mecanismo subyacente estaría implicado un incremento del tono dopaminérgico del sistema túbero- infundibular o bien un cambio a nivel del

Tabla 3. Valores basales de hormonas tiroideas y de la excreción urinaria de iodo/24 horas en sujetos normales de poblaciones de diferentes alturas del Perú.

\begin{tabular}{|c|c|c|c|c|}
\hline Lugar & $\mathrm{T} 4 \mu \mathrm{g} / \mathrm{dL}$ & $\mathrm{T} 3 \mathrm{ng} / \mathrm{dL}$ & TSH uUi/mL & EUI $\mu \mathrm{g} / 24 \mathrm{~h}$ \\
\hline $\begin{array}{c}\text { Lima }(N=10) \\
150 \text { m.s.n.m } \\
X \pm \text { E.S. }\end{array}$ & $8,4 \pm 0,3$ & $144,2 \pm 10,8$ & $3,1 \pm 0,7$ & $345 \pm 28$ \\
\hline $\begin{array}{c}\text { Tarma }(N=10) \\
3000 \text { m.s.n.m. } \\
X \pm \text { E.S. }\end{array}$ & $7,9 \pm 0,5$ & $129,3 \pm 12,2$ & $4,7 \pm 1,4$ & $129 \pm 40$ \\
\hline $\begin{array}{c}\text { Cerro de Pasco }(\mathrm{N}=11) \\
4200 \text { m.s.n.m. } \\
\mathrm{X} \pm \text { E.S. }\end{array}$ & $7,6 \pm 0,4$ & $168,1 \pm 21,1$ & $2,9 \pm 0,6$ & $228 \pm 50$ \\
\hline
\end{tabular}

P no significativo en las diferentes comparaciones de T3, T4 y TSH.

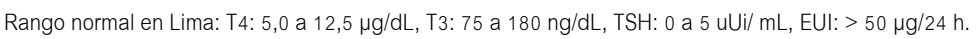


receptor o post-receptor de TRH en el lactotrofo y podría ser un mecanismo de adaptación para la conservación de la fertilidad en los hombres normales de las grandes alturas. Es importante tener en cuenta estos hallazgos para el diagnóstico y manejo de la hiperprolactinemia en los humanos de las grandes alturas.

\section{REFERENCIAS BIBLIOGRÁFICAS}

1. Gonzales GF, Kaneku L, Góñez C. Fisiologia y patología de la reproducción de las grandes alturas. Acta Andina. 1992;1:3 -16.

2. Rastogui GK, Malhorta MS. Study of the pituitary thyroid function at high altitude in man. $\mathrm{J}$ Clin Endocr Metab. 1977; 44:447.

3. Moncloa F, Guerra-Garcia R, Subauste C, Sobrevilla L, Donayre J. Endocrine studies at high altitude. I. Thyroid function in sea-level natives exposed for two weeks at altitude of 4,300 meters. J Clin Endocr Metab. 1966;26:1237.

4. Surks MI, Beckwith HJ, Chidsey Ch. Changes in plasma thyroxine concentration and metabolism, catecholamine excretion and basal $\mathrm{O} 2$ consumption in man during acute exposure to high altitude. J Clin Endocr Metab. 1967;27:789.

5. Pretell E, Garcia MA, Guerra-Garcia R, Wam M. Función tiroidea en condiciones de hipoxia crónica en nativos normales de altura (NNA) y con mal de montaña crónico (MMC) a 4,250 m.s.n.m. V Jornadas Peruanas de Endocrinología, Cusco - Perú. 1973; Abst. 26:44.

6. Sutton J, Young J, Lazarew L, Hickie JB, Garmendia $F$, Velásquez $T$. The hormonal response to high altitude. Lancet. 1970;2:1194.

7. Villena A. Características endocrino metabólicas de ancianos varones de la ciudad de Cusco $(3,400$ m.s.n.m.) y de Lima (150 m.s.n.m) Abst. 42. Acta Andina. 1992;1:45.

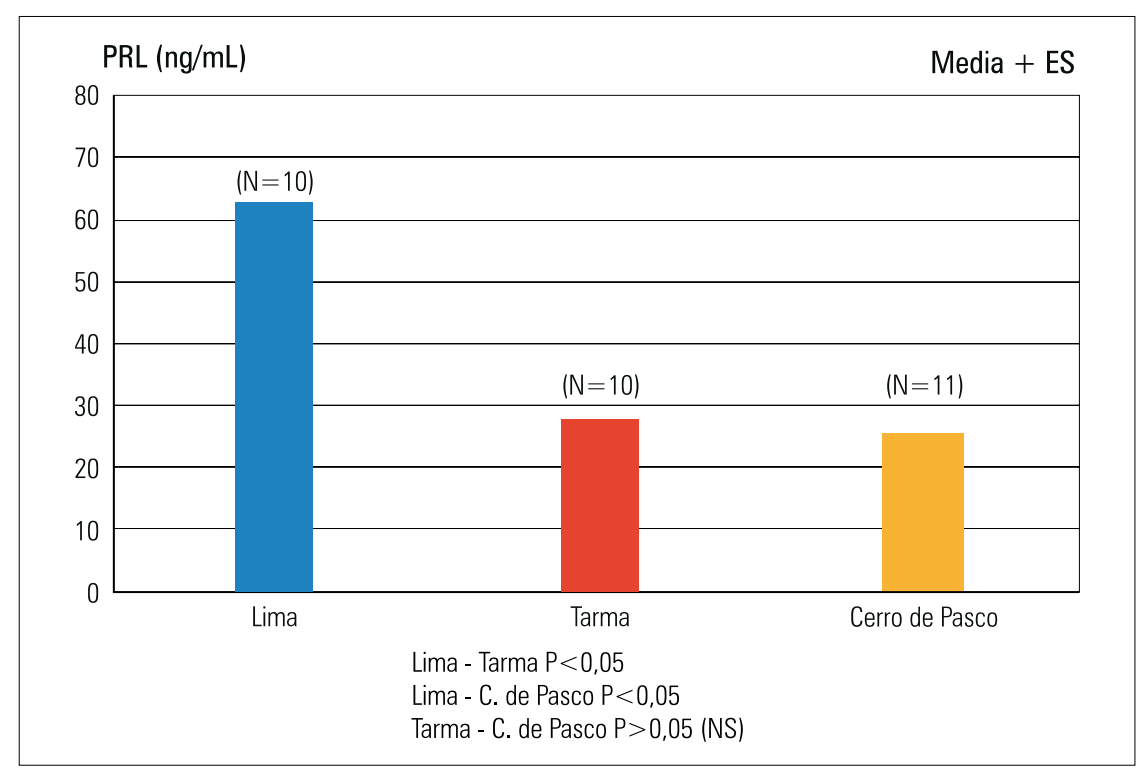

Figura 3. $\Delta$ máximo de prolactina post $200 \mu \mathrm{g}$ de TRH.

8. Pretell E. Función tiroidea en condiciones ambientales de altura Abst. 44. Acta Andina. 1992;1:46.

9. Solís J, Guerra-García R, Acosta S, Hurtado J, Vásquez S, Alarcón R. Prolactina en condición basal y después de la inyección endovenosa de TRH en sujetos normales de poblaciones de diferentes alturas del Perú. XI Jornadas Peruanas de Endocrinologia, Huancayo- Perú 1981.

10. Quintana L, Coyotupa J, Ramírez T, Carrillo C, Gonzales CF. Prolactina en mujeres de altura y del nivel del mar. Ginecol Obstet (Perú). 1991;37:88-94.

11. Lasern PR. Immunoassay of thyroxine in unextracted human serum. J Clin Endocr Metab. 1973;37:177.
12. Chopra IJ, Salomon DH, Baall GH. Radioimmunoassay for measurement of triiodothyronine in human serum. J Clin Invest. 1971;50:2033-41.

13. Odell WD, Wilber JF, Paul WR. RIA of thyrotropin in serum. J Clin Endocr Metab. 1965;23:47.

14. Sinha YN, Selby FW, Lewis VJS, Vanderhan WP. Homologous RIA for human prolactin. J Clin Endocr Metab. 1973;36:509.

15. Documenta Geigy - Tablas Cientificas: Métodos estadisticos. Sexta Edición. 1965:147-200.

16. Benotti J, Benotti N. Protein bound iodine, total iodine and butanol extractable iodine by partial automation. Clin Chem. 1963;9:408-16.

Tabla 4. Hormonas tiroideas y PRL en condición basal y post TRH (200 $\mu$ g E.V.) en tres pacientes con mal de montaña crónica, nativos de Cerro de Pasco (4 200 m.s.n.m.), en comparación con nativos normales del mismo lugar.

\begin{tabular}{|c|c|c|c|c|c|c|c|c|c|c|}
\hline \multirow{2}{*}{ Sujeto } & \multirow{2}{*}{$\mathrm{T} 4 \mu \mathrm{g} / \mathrm{dL}$} & \multirow{2}{*}{$\mathrm{T} 3 \mathrm{ng} / \mathrm{dL}$} & \multirow{2}{*}{ TSH uui/mL } & \multicolumn{7}{|c|}{ PRL (ng/mL) } \\
\hline & & & & $0^{\prime}$ & $15^{\prime}$ & $30^{\prime}$ & $60^{\prime}$ & $90^{\prime}$ & $120^{\prime}$ & $180^{\prime}$ \\
\hline $\begin{array}{l}\text { A.H.M. } \\
\text { HTC: } 69\end{array}$ & 5,8 & 71 & 2 & 2,6 & 3,4 & 12,1 & 26 & 3,1 & 5,6 & 5,6 \\
\hline $\begin{array}{l}\text { R.S.M. } \\
\text { HTC: } 63\end{array}$ & 8,1 & 100 & 2,5 & 3,3 & 17,8 & 21,5 & 24 & 6,6 & 4,7 & 2,9 \\
\hline $\begin{array}{c}\text { E.P. } \\
\text { HTC: } 67\end{array}$ & 6,1 & 112 & 1 & 5,9 & 21,5 & 34 & 10,2 & 6,2 & & \\
\hline $\begin{array}{c}\text { Suj. normales Cerro } \\
\text { de Pasco } \\
\text { X+E.S. } \\
\text { (N=11) } \\
\text { EUI: } 228+50 \\
\text { HTC: } 54,3\end{array}$ & $\begin{array}{l}7,6 \\
+- \\
0,4\end{array}$ & $\begin{array}{c}168,1 \\
+- \\
21,1\end{array}$ & $\begin{array}{c}2,9 \\
+- \\
0,62\end{array}$ & $\begin{array}{c}7,67 \\
+- \\
0,96\end{array}$ & $\begin{array}{c}32,94 \\
+- \\
4,87\end{array}$ & $\begin{array}{c}29,3 \\
+- \\
2,9\end{array}$ & $\begin{array}{c}21,9 \\
+- \\
2,7\end{array}$ & $\begin{array}{c}12,84 \\
+- \\
2,5\end{array}$ & $\begin{array}{c}10,9 \\
+- \\
2,5\end{array}$ & $\begin{array}{l}8,5 \\
+- \\
1,74\end{array}$ \\
\hline
\end{tabular}

$\mathrm{HTC}=$ hematocrito. $\mathrm{EUI}=$ excreción urinaria de iodo. 
17. Solis J, Cornejo P. Estados hiperprolactinémicos. Rev Med Hered. 2006;17:234-45.

18. Solis J. Función tiroidea y secreción de prolactina en varones normales en situaciones de hipoxia crónica. 1992. Tesis Doctoral de la Universidad Peruana Cayetano Heredia.

19. Llaque W. Estudio del eje hipotálamo-hipófisisgonadal en hombres normales a nivel del mar y en la altura. Tesis Doctoral. Universidad Peruana Cayetano Heredia. 1974, Lima- Perú.

20. Coyotupa J, Llerena LA, Guerra-Garcia R. Respuesta a Gn-Rh en hombres normales de altura y del nive del mar. VII Jornadas Peruana de Endocrinologia, Ica - Perú. 1977; Abst. 42.

21. Fink G. Neuroendocrine control of gonadotrophin secretion. Br Med Bull. 1979;35:155.
22. Barraclough CA, Wise PM. The role of catecholamines in the regulation of pituitary luteinizing hormone and follicle-stimulating hormone secretion. Endocr Rev. 1982;3:91.

23. Ingbar SH. The Thyroid Gland: Textbook of Endocrinology: Wilson-Foster (Ed.) Seventh edition. W.B. Saunders Company. 1985;628-815.

24. Yen SSC. Neuroendocrine regulation of gonadotropin and prolactin secretion in women: Disorders in reproduction. En: Vaitukaitis $\mathrm{JL}(\mathrm{Ed})$. Current Endocrinology, Clinical Reproductive Neuroendocrinology Section. New York: Elsevier Biomedical. 1982:137-76.

25. Gibs DM, Neil JD. Dopamine levels in hypophyseal stalk blood in the rat are sufficient to inhibit prolactin secretion in vivo. Endocrinol. 1978;102:1895.
26. Garmendia F, Castillo O, Valdivia M. Sensibilidad hipófiso-testicular del nativo normal de altura a la administración de hormona liberadora de gonadotropinas. Arch Biol Andina. 1984-1985;13:207-11.

27. Garmendia F, Castillo O, Ugarte N, Garmendia A Hypothalamus-hypophysis-gonadal response to clomiphene citrate at median high altitude. Horm Metab Res. 1982;14:679-80.

Trabajo recibido el 6 de octubre de 2010 y aceptado para publicación el 7 de diciembre de 2010. 